\title{
Concept Level Evaluation of the Optical Voltage and Current Sensors and an Arrayed Waveguide Grating for Aero-Electrical Systems' Applications
}

\author{
G. Fusiek, P. Niewczas, J. R. McDonald \\ Institute for Energy and Environment, Dept. of Electronic and Electrical Eng., University of Strathclyde, Glasgow, UK \\ Phone: +44 (0)141 548 4841, Fax: +44 (0)141 5484872 \\ Email: gfusiek@eee.strath.ac.uk \\ URL: http://www.isap.eee.strath.ac.uk
}

\begin{abstract}
In this paper we present, for the first time, the hybrid fiber-optic voltage and current sensors interrogated using an Arrayed Waveguide Grating ( $A W G$ ) device. Due to the excellent dynamic capabilities of an AWG-based interrogator and its improved robustness, the proposed system would be suitable for voltage and current monitoring within an aircraft electrical system. The voltage sensor comprises a multilayer piezoelectric stack, acting as a voltage-to-strain transducer, and a fiber Bragg grating (FBG) used to convert voltage induced strain changes within the stack into wavelength shifts. These wavelength shifts are then analysed by an $A W G$. To measure current, the same sensor type is used to monitor a specially designed ferrite-core current transformer. It is shown that the system is capable of measuring variable frequency of voltage and current waveforms, typical of those anticipated in the next generation aero-electric power systems. It is also demonstrated that the system can be used for voltage and current harmonic analysis and power quality measurement in such networks.
\end{abstract}

Kevwords - Fiber Bragg Grating sensors, piezoelectric transducers, arrayed waveguide gratings, optical voltage sensors, optical current sensors, aircraft electrical system.

\section{INTRODUCTION}

It is of great importance to measure and monitor voltage and current levels as well as voltage and current waveforms within the aircraft electrical system. The main reasons are to provide information to fault detection and protection systems and for diagnostic purposes. These unique in their nature electrical systems incorporate both single and three-phase distribution networks, whilst electrical loads operate on different voltages (both ac and dc). The systems utilize $28 \mathrm{~V}$ $\mathrm{dc}$ power sources and engine-driven generators that produce voltage of $115 \mathrm{~V} \mathrm{ac}, 400 \mathrm{~Hz}$. There are also systems equipped in $270 \mathrm{~V} \mathrm{dc}$ power sources and variable-frequency power generators with frequency ranges from 200 to $800 \mathrm{~Hz}$. Standard aircraft loads and normal operating conditions such as motor start-ups, strobe and landing lights, bus transfers, fluorescent lighting loads or opening and closing of mechanical switches can generate undesirable transient conditions. Many of the loads and transient conditions can exhibit signatures that can be symptomatic of an arcing condition. This signature information can be used for the purpose of protection against the arc fault condition. Therefore, it is of great importance to monitor voltage and current waveforms of different amplitudes and frequencies within aircraft electrical systems as, when appropriately analyzed, they can be used to detect the arcing conditions and other anomalies [1].

Currently, these measurements are performed using current and voltage instrument transformers. However, with an increased complexity of the electrical network in a commercial aircraft, the weight associated with the cabling is significant. Every additional kilogram added to the aircraft weight cannot be utilized for the normal load carrying purposes; every additional kilogram added to the aircraft weight causes increased emissions. Therefore, it is desirable to reduce the weight of all the systems within the airframe. However this should be achieved without sacrificing the functionality and safety.

To achieve the weight reduction of aircraft instrumentation systems, this work proposes that optical sensors are used for measuring voltage and current, and, in particular, fiber Bragg grating based sensors which can be multiplexed [2]-[4]. Optical sensors due to their light weight, small size, immunity to electromagnetic interference, and intrinsically safe mode of operation would be extremely valuable for such an application.

Previously we proposed hybrid voltage and current sensors for remote condition monitoring of electrical submersible pumps (ESPs) (see Figure 1) [5], [6]. We also proposed an interrogation system for this application based on a FabryPerot tunable filter [7]. The complete system was capable of measuring dynamically changing signals and was successfully used for detecting higher order voltage and current harmonics [7], [8].

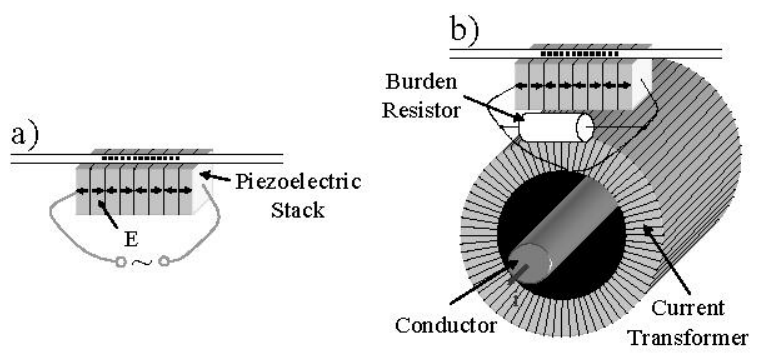

Figure 1. a) Hybrid voltage sensor; b) Current sensor employing a voltage sensor and a current transformer. 
In this paper, we carry out a concept level evaluation of the optical sensor system for the purpose of aircraft electrical system monitoring. An Arrayed Waveguide Grating (AWG) device is proposed as an interrogator for the hybrid voltage and current sensors. It is hoped that this solid-state component would provide the necessary robustness of the system in this safety critical application and would offer superior dynamic capabilities for measuring voltage and current waveforms over a wide range of frequencies.

\section{MEASUREMENT SYSTEM}

The application of an AWG as an interrogator of serially arranged FBGs has already been reported in the literature [4], [9]-[13]. These systems use pairs of AWG channels to derive ratiometric transfer functions of the individual FBGs. As shown in Figure 2, depending on the spectral position of the reflected FBG peak between the pair of the AWG channels, different optical power is transmitted through the channels. Providing that the sensing FBG peak is placed centrally between the two adjacent AWG channels, this allows for measuring the FBG peak position.

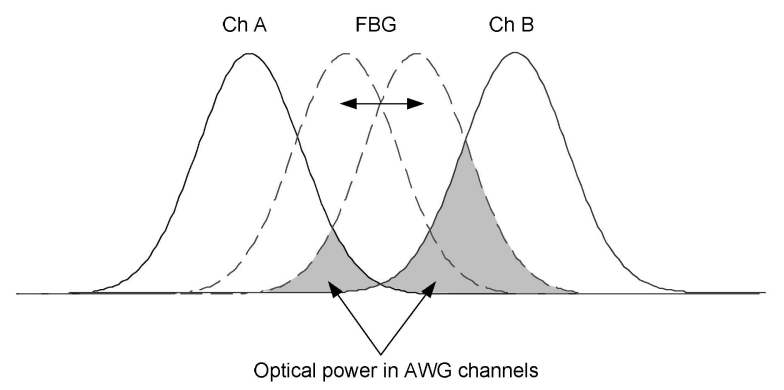

Figure 2. Optical power distribution between two adjacent channels of an AWG.

To quantify the sensor response we calculated the transfer function according to the equation:

$\mathrm{SR}=\frac{\mathrm{Ch} A-\mathrm{Ch} \mathrm{B}}{\mathrm{Ch} \mathrm{A}+\mathrm{Ch} \mathrm{B}}$

where SR is the sensor response, Ch A and Ch B are optical powers transmitted through respective channels or equivalent photodetectors output voltages.

As the FBG peak position is modulated across the AWG channels the sensor response described by (1) changes as shown in Figure 3. The grey region in the figure highlights the linear sensor response.

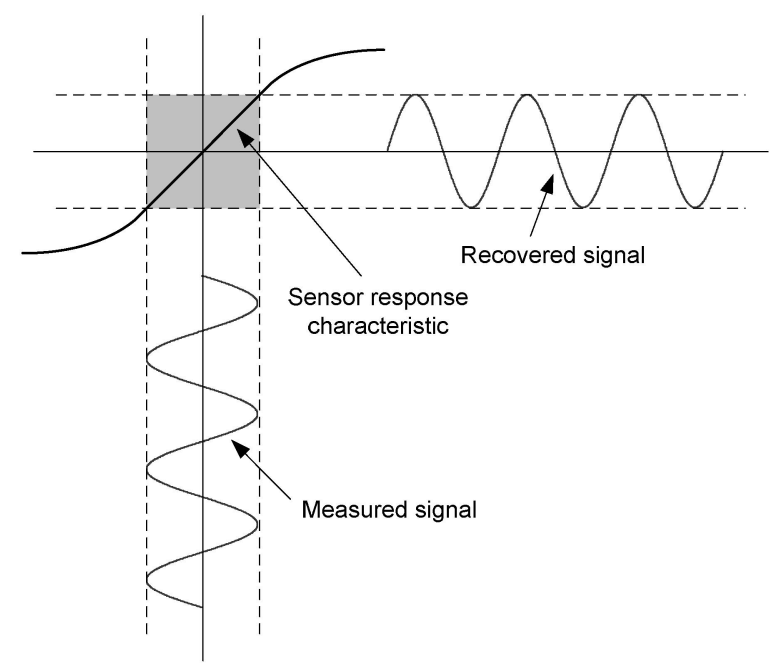

Figure 3. Illustration of the measured signal recovery.

For the method to be suitable for the interrogating the hybrid voltage and current sensors the following conditions have to be met:

- reflection peak of the sensing FBG must fall centrally between the individual pair of the AWG channels;

- FBG and AWG channels bandwidths must be matched to provide sufficient optical signal recovery;

- maximum operating wavelength range of the sensors must be within the linear part of the system response characteristic.

These conditions can be satisfied upon the careful voltage and current sensors designs.

\section{EXPERIMENTAL RESULTS}

In order to demonstrate in practice the system performance and the possibility of measuring voltage and current waveforms the respective experimental set-ups were employed as shown in Figure 4 and Figure 5. A common part of both set-ups was the interrogation system. This involved a broadband super fluorescent source (SFS), having a $3 \mathrm{~dB}$ wavelength range of $1525-1565 \mathrm{~nm}$, which was used to illuminate the hybrid FBG sensor (bandwidth of $0.3 \mathrm{~nm}$, centre wavelength of $1546 \mathrm{~nm}$ ) through an optical coupler and a given length of optical fiber. A 16-channel, $100 \mathrm{GHz}$ spacing AWG device was used as a spectrum analyzer. The signal reflected from the sensor was recorded using two photodetectors and a PXI data acquisition card (National Instruments), and subsequently processed in real time to recover the measured signal. The AWG channels' positions were adjusted and stabilized using a thermoelectric cooler (TEC) controller to match the hybrid sensor FBG peak position. All optical components were interconnected using single-mode fiber, spliced or terminated with angle polished contact connectors.

A state-of-the-art power signal generator (APTS3AI, Relay Engineering Services Ltd) was used to test the sensors. The device allowed for generating sine-wave signals having 
amplitude up to $600 \mathrm{~V}$ rms and frequency up to $1 \mathrm{kHz}$. In addition, by generating $50-\mathrm{Hz}$ voltage and current square waveforms, a wide range of harmonics content could be provided, limited only by the signal generator's bandwidth, which was $4 \mathrm{kHz}$ ( $3 \mathrm{~dB}$ cut-off point).

Voltage across the electrical terminals of the voltage sensor was measured using a PXI data acquisition card, connected through a $100 \mathrm{~V} / 1 \mathrm{~V}$ ratio voltage divider and a buffer amplifier (Figure 4). The buffer amplifier was used for the purpose of impedance matching between the voltage divider and the PXI card.

The current sensor was connected in series with a $0.1 \Omega$ load resistor and the APTS signal generator (Figure 5). Voltage across the resistor was measured using the PXI data acquisition card and calibrated to give reference current readings.

All measurements were performed at the sampling rate of $10 \mathrm{kSps}$.

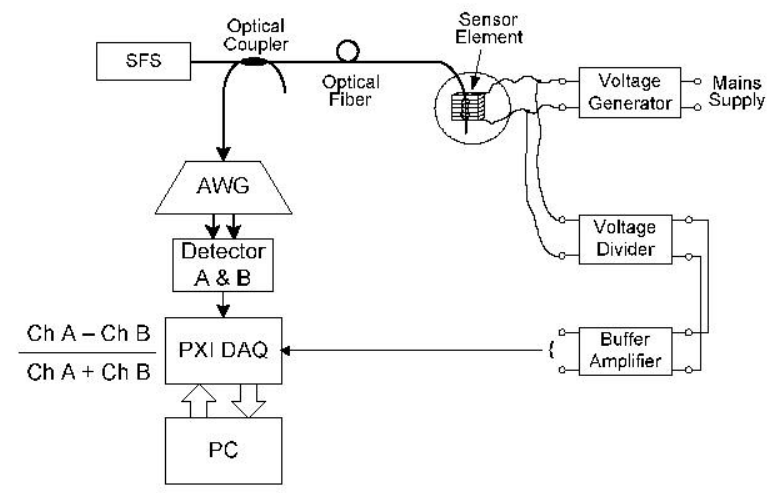

Figure 4. Experimental set-up for voltage characterization.

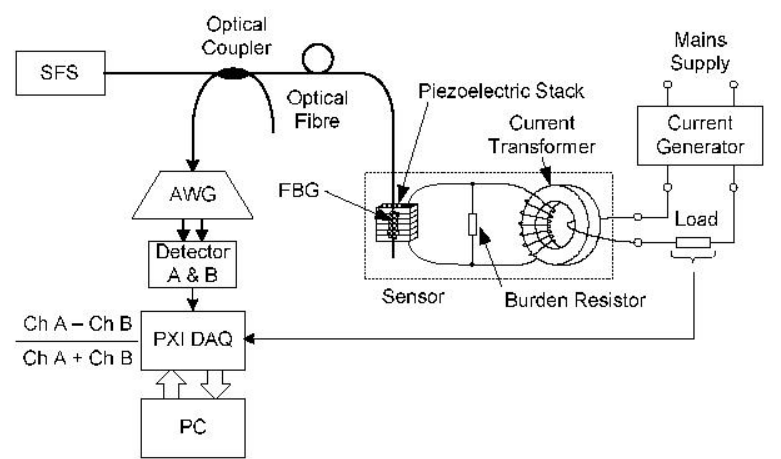

Figure 5. Experimental set-up for current characterization.

The first experiment involved the sensor response/ AWG interrogator characterisation (see Figure 6). Dc voltage was applied to the voltage sensor to sweep the FBG peak over the pair of the AWG channels and the sensor response was expressed in terms of wavelength. As marked in the figure, the sensor response was linear up to approximately $200 \mathrm{~V}$ rms.

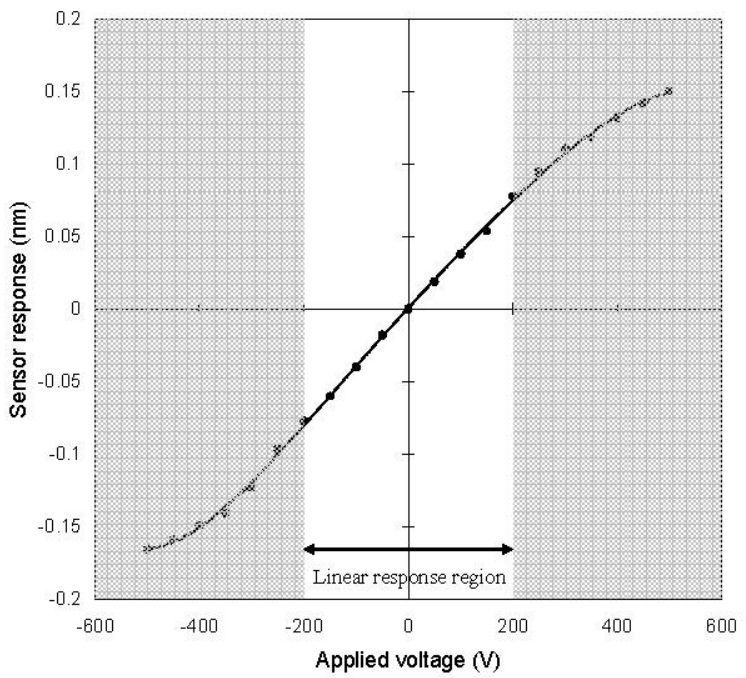

Figure 6. Sensor response characteristic.

The sample experimental results of voltage and current measurements are shown in Figure 7 through to Figure 14. Figure 7 and Figure 8 show the voltage measurement results of the $50-\mathrm{Hz}$ and $1-\mathrm{kHz}$ sine-waves respectively. Figure 9 and Figure 10 depict $50-\mathrm{Hz}$ square-wave voltage recovery and Fast Fourier Transform (FFT) analysis, respectively. As can be seen from the figures, the voltage traces and their respective harmonic content are reproduced with high fidelity by the optical sensor. In Figure 10 the optical signal was normalized to the fundamental harmonic of the reference signal.

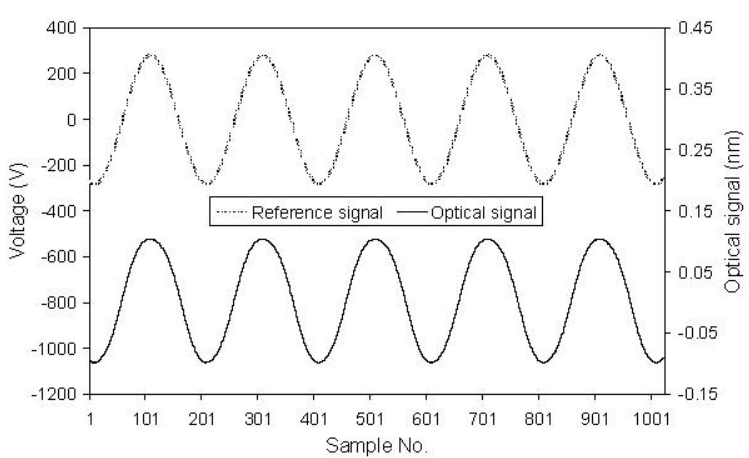

Figure 7. Voltage sensor response to $50 \mathrm{~Hz}$ sine-wave. 


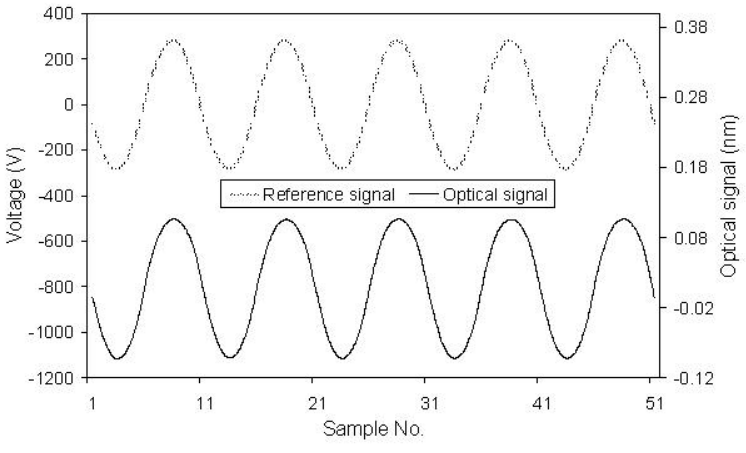

Figure 8. Voltage sensor response to $1 \mathrm{kHz}$ sine-wave.

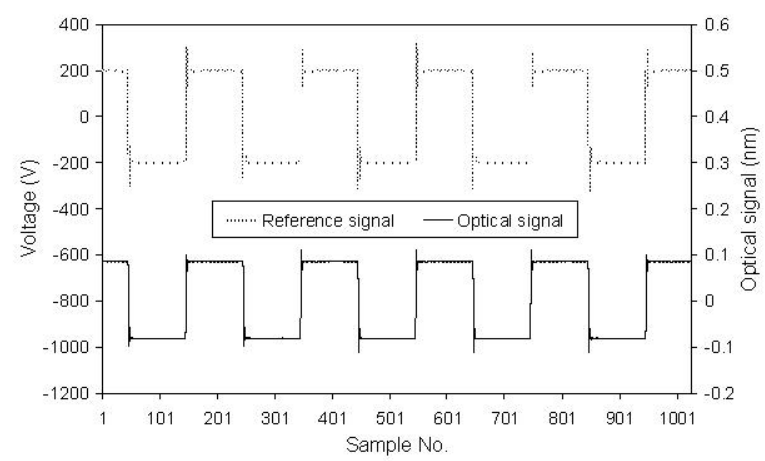

Figure 9. Voltage sensor response to $50 \mathrm{~Hz}$ squre-wave.

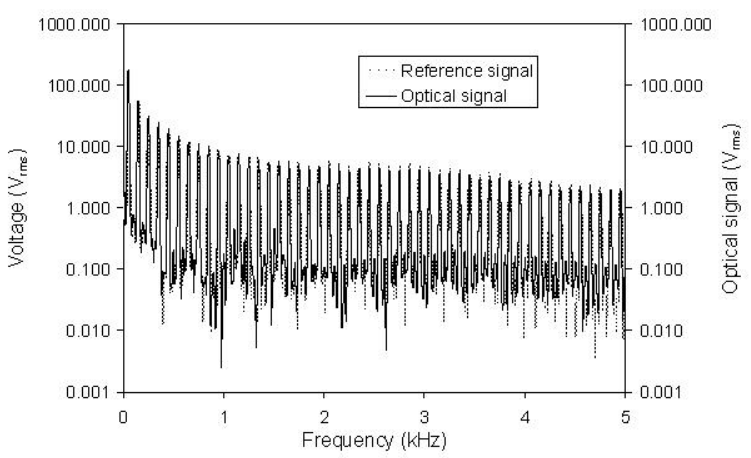

Figure 10. FFT of the voltage reference and optical signals at $200 \mathrm{~V}$.

Similar experiment was performed with the hybrid current sensor. Figure 11 and Figure 12 show the results of the current measurement of the $50-\mathrm{Hz}$ and $1-\mathrm{kHz}$ sine-waves respectively. Figure 13 and Figure 14 depict the results when a $50-\mathrm{Hz}$ square-wave current waveform was applied to the sensor and its FFT analysis, respectively. Due to the high impedance of the special high-output-voltage current transformer, the current waveforms are distorted to some extent (see Figure 13). However, the sensor can still be valuable in detecting higher order harmonics of the current waveform as the harmonic contents of the reference and optical signals are largely in agreement with each other (see Figure 14). Again, the optical signal in Figure 14 was normalized to the fundamental harmonic of the reference signal.

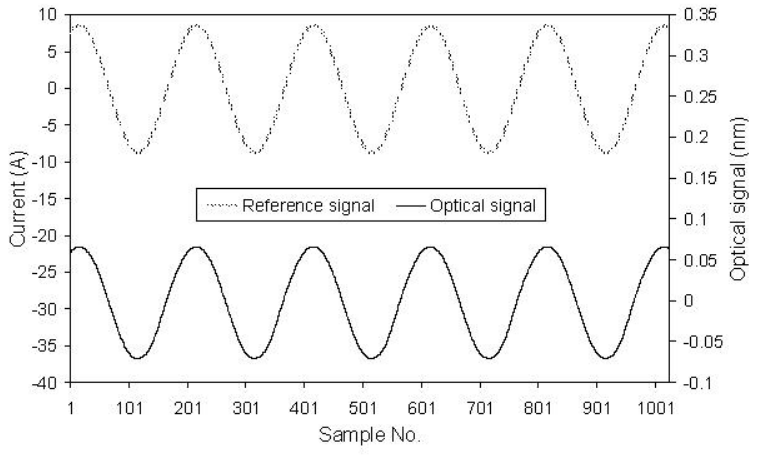

Figure 11. Current sensor response to $50 \mathrm{~Hz}$ sine-wave.

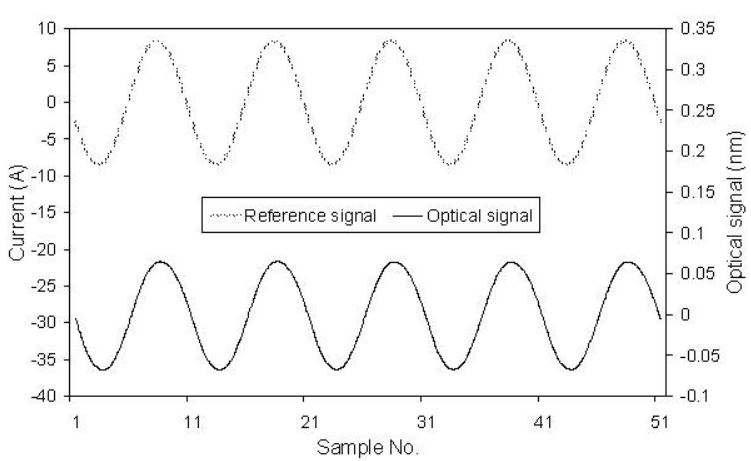

Figure 12. Current sensor response to $1 \mathrm{kHz}$ sine-wave.

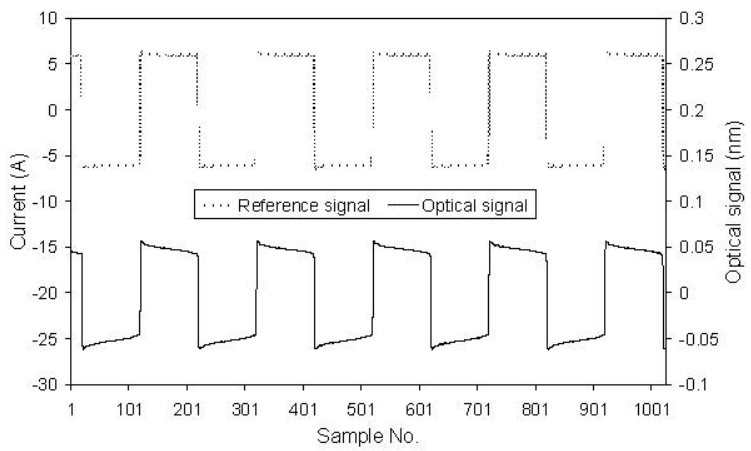

Figure 13. Current sensor response to $50 \mathrm{~Hz}$ square-wave having amplitude of $6 \mathrm{~A}$.

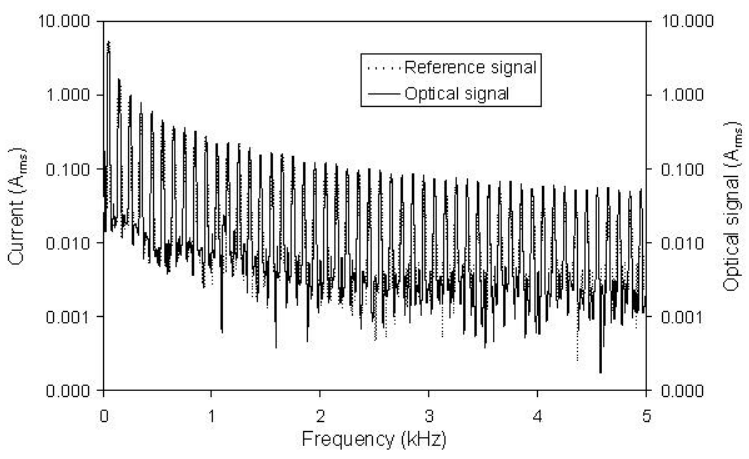

Figure 14. FFT of the current reference and optical signals at $6 \mathrm{~A}$. 
To determine the resolution of the system, its response was recorded at the absence of the input voltage or current signals. The system noise shown in Figure 15 includes sources of amplitude noise such as the photodetector noise, quantization noise and signal processing errors.

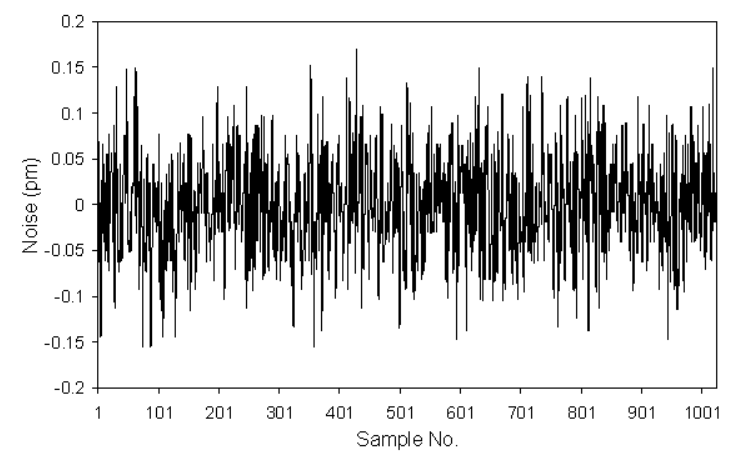

Figure 15. Noise of the AWG-based interrogation system.

As can be seen from the figure, the resolution of the system is approximately $0.15 \mathrm{pm}$, which gives signal-to-noise ratio (SNR) of approximately $55 \mathrm{~dB}$ at $200 \mathrm{~V}$ input signal. This is a considerable improvement in comparison to commercially available interrogation systems with typical resolution of $1 \mathrm{pm}$.

\section{CONCLUSIONS}

In this paper we have demonstrated the successful implementation of an arrayed waveguide grating (AWG) as a solid-state interrogation component for demodulating hybrid fiber-optic voltage and current sensors. We have shown that the use of an AWG brings the benefits of the improved SNR and greater frequency of operation in comparison with alternative interrogation techniques. As a consequence, it is possible to apply harmonic analysis to the measured voltage and current waveforms in order to identify various anomalies of the electrical network. The improved performance and potentially reduced weight of the optical voltage and current measurement system in comparison with conventional electrical instruments render this measurement method particularly suitable for aero-electrical applications.

\section{REFERENCES}

[1] T. E. Potter, "Arc fault Interruption Requirements for Aircraft Applications", Texas Instruments, online: http://www.sensata.com,

[2] Y. J. Rao, "In Fibre Bragg grating sensors," Meas. Sc. Tech. 8 (1997) 355-375,

[3] A. D. Kersey, M. A. Davis, H. J. Patrick, M. LeBlanc, K. P. Koo, C. G. Askins, M. A. Putnam and E. J. Friebele, "Fiber Grating Sensors", Journal of Lightwave Technology, Vol. 15, No. 8, August 1997 ,

[4] P. Niewczas, A. J. Willshire, L. Dziuda, J. R. McDonald, "Performance analysis of the fibre Bragg grating interrogation system based on an arrayed waveguide grating", IEEE Transactions on Instrumentation and Measurement, 53 (4): 1192 - 1196, August 2004
[5] L. Dziuda, P. Niewczas, G. Fusiek, J. R. McDonald, "Hybrid FiberOptic Voltage Sensor for Remote Monitoring of Electrical submersible Pump Motors", Optical Engineering, Vol. 44, No. 6, pp 64401-1-6, June 2005

[6] L. Dziuda, P. Niewczas and J.R. McDonald, "Hybrid Fiber-Optic Current Sensor for Remote Monitoring of Electrical Submersible Plant", Proceedings of Sensors '05 Conference, October 2005

[7] G. Fusiek, P. Niewczas, J. R. McDonald, "Extended Step-out Length Fiber Bragg Grating Interrogation System for Condition Monitoring of Electrical Submersible Pumps", Optical Engineering, Vol. 44, No. 3, pp 034404-1-10, March 2005

[8] P. Niewczas, G. Fusiek, J. R. McDonald, "Dynamic capabilities of the hybrid fiber-optic voltage and current sensors", IEEE Sensors Conference, Daegu, Korea, Oct. 22-25, 2006

[9] Y. Sano, N. Hirayama, T. Yoshino, "Wavelength interrogator employing arrayed waveguide grating for distributed fiber Bragg grating sensors", 14th International Conference On Optical Fiber Sensors, Proceedings Of The Society Of Photo-Optical Instrumentation Engineers (SPIE), 4185, 788-791, 2000.

[10] Y. Sano and T.Yoshino, "Fast optical wavelength interrogator employing arrayed waveguide grating for distributed fiber Bragg grating sensors," J. Lightw. Technol, vol. 21, no. 1, pp. 132-139, Jan. 2003.

[11] G. Z. Xiao, P. Zhao, F. Sun, Z. Lu, Z. Zhang, "Arrayed-waveguidegrating-based interrogator for wavelength-modulated multi-fiber-optic sensor applications", EEE Photonics Technology Letters, Vol. 17, No. 8, pp. 1710-1712, August 2005

[12] D. C. C. Norman, D. J. Webb, and R. D. Pechstedt, "Extended range interrogation of wavelength division multiplexed fiber Bragg grating sensors using arrayed waveguide grating," Electron. Lett., vol. 39, pp. $1714-1715,2003$

[13] D. C. C. Norman, D. J. Webb, R. D. Pechstedt, "Interrogation of fibre Bragg grating sensors using an arrayed waveguide grating", Meas. Sci. Technol. 16 (2005) 691-698. 